\title{
ChemComm
}

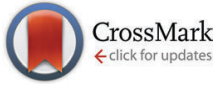

Cite this: Chem. Commun., 2015, 51, 7015

Received 16th February 2015 Accepted 9th March 2015

DOI: $10.1039 / c 5 c c 01445 j$

www.rsc.org/chemcomm

\section{Probing extracellular acidity of live cells in real time for cancer detection and monitoring anti-cancer drug activity $\dagger$}

\author{
Bhawana Thakur, ${ }^{a}$ S. Jayakumar ${ }^{\mathrm{b}}$ and Shilpa N. Sawant*a
}

\begin{abstract}
We report a novel electrochemical strategy to probe the microenvironment of live cells in real time in terms of its extracellular $\mathrm{pH}$. This approach allowed highly sensitive detection of cancer cells down to five cells. Utility of the sensor for evaluating the efficacy of glycolysis inhibiting anti-cancer drugs is also demonstrated.
\end{abstract}

Cancer is one of the major health challenges worldwide. Its detection in the early stage is crucial for an effective treatment. The conventional technique for detection of cancer is based on histopathology, which involves microscopic examination of the biopsy sample. Histopathology requires tedious sample preparation steps, which span over several hours. ${ }^{1}$ Moreover, trained pathologists are required for sample examination and interpretation. The other method commonly used is the estimation of biomarkers such as proteins, nucleic acids or hormones. ${ }^{2}$ However, low expression of biomarkers poses a limit on detection of cancer in the early stages. Hence, there is a need for a low cost, simple detection technique for initial screening of tumor samples irrespective of their origin. It is desirable to rely on a common biomarker or some inherent physiological properties, which is a signature of the cancer cell in general.

A universal property of primary and metastatic cancers is upregulation of glycolysis resulting in increased glucose consumption, which has been confirmed by positron emission tomography. ${ }^{3}$ Both primary and malignant lesions were found to consume glucose at a high rate irrespective of the oxygen status. The abnormal metabolism exhibited by cancer cells is characterized by excessive glycolysis, which leads to conversion of glucose to lactic acid (Warburg effect). ${ }^{4}$ The extent to which this phenomenon is expressed correlates with the tumor aggressiveness. Due to excessive production of lactic acid, the

\footnotetext{
${ }^{a}$ Chemistry Division, Mumbai 400085, India

${ }^{b}$ Radiation Biology \& Health Sciences Division, Bhabha Atomic Research Centre, Mumbai 400085, India. E-mail: stawde@barc.gov.in; Tel: +91-22-25590288 $\dagger$ Electronic supplementary information (ESI) available. See DOI: 10.1039/ c5 5 c01445j
}

extracellular microenvironment of most tumors is found to be mildly acidic. ${ }^{5}$ Thus, tumors exhibit a substantially lower extra cellular $\mathrm{pH}\left(\mathrm{pH}_{\mathrm{e}}\right)$ than normal tissues, whereas the intracellular $\mathrm{pH}$ of both tissues is $\operatorname{similar}^{6}$ (by virtue of proton pumps and intracellular buffers). This $\mathrm{pH}$ difference is exploited to a large extent for diagnosis and therapy by the design and use of $\mathrm{pH}$ sensitive agents that target the acidic tumor site. Studies by Lindner et $a .^{7}$ reveal that $\mathrm{pH}_{\mathrm{e}}$ based studies can help to determine the efficacy of various non-surgical therapies, such as irradiation, chemotherapy and hyperthermia, and have significant impact on the management of cancer. ${ }^{8}$ In spite of its clinical significance, there are limited approaches (such as MRI, PET etc.; ESI, $\dagger$ Section 1a) for rapid, simple and low cost measurement of local $\mathrm{pH}$ which can facilitate early prediction in clinical settings.

Herein, we demonstrate for the first time, an electrochemical approach for real-time monitoring of the extracellular acidity $\left(\mathrm{pH}_{\mathrm{e}}\right)$ in the microenvironment of live cells. As the $\mathrm{pH}_{\mathrm{e}}$ values are correlated with the metabolic state of the cells, the sensor could probe the abnormal metabolism exhibited by the cancer cells thus corroborating its utility in potential clinical application for detection of cancer. By ingenious design of the electrodes, it was possible to achieve highly sensitive detection of as low as five cancer cells. The sensor is further utilized to evaluate the efficacy of glycolysis inhibiting drugs used in cancer therapy.

Our approach involves use of a conducting polymer, polyaniline, as the active platform for sensor development due to its high sensitivity to gauge $\mathrm{pH}$ change in its microenvironment, especially during the course of the biochemical process as corroborated in one of our earlier studies on detection of pesticide lindane at ppt levels. ${ }^{9}$ In the present case, the electrochemical transduction involves doping of polyaniline by the acidic metabolites released by the cells in their microenvironment upon glycolysis and the amperometric detection at an optimized voltage of $0.4 \mathrm{~V}$. The acidic metabolites produced by the cells which are in direct contact with the sensor surface leads to conversion of the emeraldine base form of polyaniline to emeraldine salt (Scheme 1). This results in an 


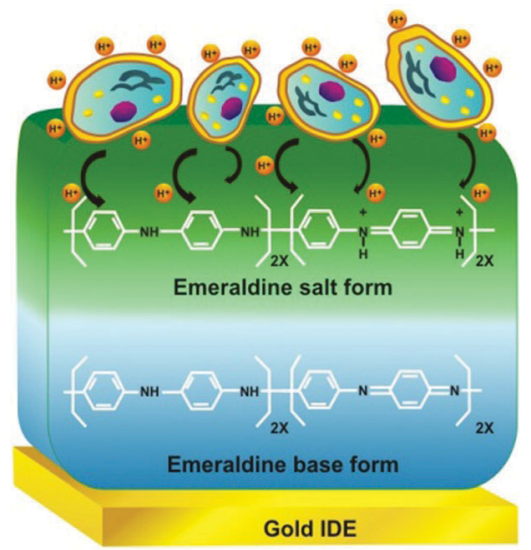

Scheme 1 The acidic metabolites produced by the cells in direct contact with the sensor surface leads to conversion of the emeraldine base form of polyaniline to emeraldine salt form with a concomitant increase in its conductivity.

increase in conductivity of the sensor film, which is measured by amperometry. We have investigated two different types of electrode (Fig. S1 in the ESI $\dagger$ ) assemblies, sensors 1 and 2. Highly sensitive detection of the cancer cells could be attained due to the exponential increase in conductivity of polyaniline upon doping with the metabolites coupled with judicious design of the electrodes and the sensor assembly.

Each sensor was first calibrated (see details in the ESI, $\dagger$ Section 2C) for $\mathrm{pH}$ measurement using PBS solutions of different pH (Fig. 1A) to obtain a calibration curve as shown in Fig. 1B. The calibration curve enables direct read-out of $\mathrm{pH}$ values of test samples from the normalized current values (ESI, $†$ Section 2C). As depicted in Fig. 1B, the increase in normalised current was more prominent at lower $\mathrm{pH}$ ( 5 to 6 ) as compared to that at higher $\mathrm{pH}$. None-the-less, the response in the higher $\mathrm{pH}$ range of 6 to 8 (inset of Fig. 1B) is good enough for sensitive determination of $\mathrm{pH}$ values in this range. As a proof of concept, three cancer cell lines namely MCF7, PC3 and DU145 and noncancerous human Peripheral Blood Mononuclear cells (PBMC) were studied. The cells were washed with PBS $(\mathrm{pH}=8)$, centrifuged and re-suspended in $50 \mu \mathrm{L} \mathrm{PBS}(\mathrm{pH}=8)$. This pretreatment and buffer washing helped to eliminate the $\mathrm{pH}$ effect due to the
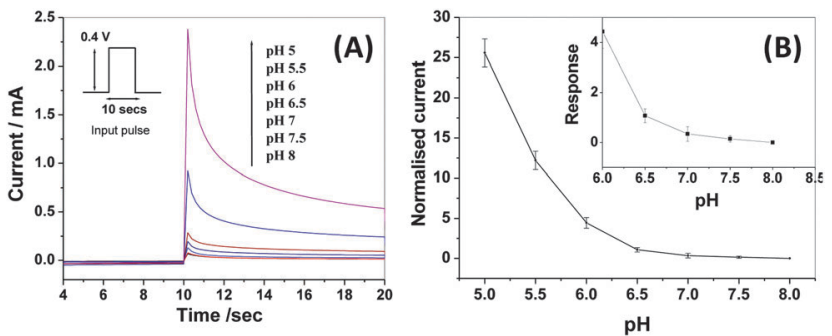

Fig. 1 (A) Potential pulse applied to sensor 1 (inset) and the resulting current obtained for PBS solutions of different $\mathrm{pH}$. (B) Normalised current response of sensor 1 to PBS solutions of different $\mathrm{pH}$ (calibration plot), inset: magnified image of the sensor response to buffer solutions in the $\mathrm{pH}$ range of $6-8(\mathrm{~N}=3)$. previous history of cell culture so that the measured $\mathrm{pH}$ has contribution only from the metabolic acids freshly generated in situ by the cells upon glycolysis.

Before starting the measurements on cancer cells, the sensor was equilibrated with a PBS solution of $\mathrm{pH} 8$ till a stable current output is obtained. Upon stabilization, a known amount of the cells under study were added to the PBS electrolyte in the sensor assembly and the resulting change in sensor response current was measured (see details in the ESI, $\dagger$ Section 2C). A representative set of amperometric responses of sensor $\mathbf{1}$ upon addition of MCF7 cells is depicted in the ESI, $\dagger$ Fig. S2A. For the various cell lines studied, the response obtained using sensor 1 at different cell densities is depicted in the ESI, $\dagger$ Fig. S2B. For each cell type studied, the response was found to rapidly increase with cell concentration up to $1 \times 10^{6}$ cells after which the increase was marginal. Since the amount of metabolic acids produced would determine the extent of doping, it was expected that the response should increase with an increase in cell density. This led us to carry out further investigation on the sensing mechanism.

The surface of the polyaniline sensor film at various cell densities was studied by fluorescence and optical microscopy (Fig. S3 and S4 in the ESI $\uparrow$ ). At a cell density of $0.5 \times 10^{6}$, the cells were found to be well separated and are able to directly interact with the polyaniline surface whereas above a cell number of $1 \times 10^{6}$, the cells begin to stack over each other. This is exactly the cell density at which the sensor response begins to saturate. Only in the case of PBMC cells, the saturation in response is observed at higher cell density probably due to its smaller size $(6-10 \mu \mathrm{m})$ as compared to the other cells (PC3 $=23 \mu \mathrm{m}$, MCF7 $=18 \mu \mathrm{m}$ ) studied. ${ }^{10}$ Thus, in the present system, the doping seems to be a surface phenomenon where only the cells directly adsorbed on the polyaniline surface are predominantly responsible for its doping and hence contribute to the sensor response. The metabolites released in the electrolyte seem to play a minor role as they get diluted and buffered by the PBS electrolyte and are also shielded from the sensor surface by the layer of adsorbed cells.

In order to corroborate the above interpretation, we designed another set of experiments where we separately studied $\mathrm{pH}$ of the supernatant solution and the cells. The cells were washed with PBS, centrifuged, re-suspended in $50 \mu \mathrm{L}$ of $\mathrm{PBS}(\mathrm{pH}=8)$, and were allowed to remain undisturbed at room temperature for one hour. After this, the cell suspension was centrifuged and the $\mathrm{pH}$ of the supernatant solution (containing the acidic metabolic products) and the precipitated cells were determined independently using the polyaniline sensor films. In the case of $1 \times 10^{6}$ MCF7 cells, the $\mathrm{pH}$ of the centrifuged cells was found to be $5.71 \pm 0.40(N=5)$ and that of the supernatant solution was found to be $6.74 \pm 0.09(N=5)$. Similarly, for $0.5 \times 10^{6}$ DU145, the $\mathrm{pH}$ of the centrifuged cells was found to be 7.37 and the $\mathrm{pH}$ of supernatant solution was found to be 7.8. The results clearly indicate that the contribution to reduction in $\mathrm{pH}$ of the sensor film is mainly from the cells as compared to the supernatant solution. Thus, the sensor is capable of measuring $\mathrm{pH}$ at the cell membrane surface. 

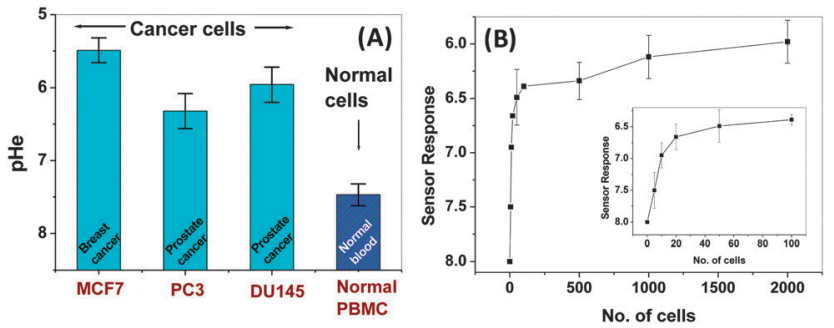

Fig. 2 (A) Response of sensor 1 to different cancerous and non-cancerous $\left(2 \times 10^{6}\right)$ cells studied (error bars correspond to $\left.N=5\right)$. (B) Response of sensor 2 to MCF7 cells, inset: magnified plot for the sensor response at a very low cell number.

The present approach thus allowed discrimination between the $\mathrm{pH}$ at the cell membrane surface vis-a-vis that in the bulk cell suspension. Zeta potential measurements were also carried out to elucidate the nature of an extracellular environment and its probable correlation with $\mathrm{pH}_{\mathrm{e}}$. The $\mathrm{pH}_{\mathrm{e}}$ values measured using the sensor were found to be independent of the charge on the cell membrane and depend on the amount of metabolites released by the cells in their microenvironment (ESI, $\dagger$ Section 3C). Thus, the $\mathrm{pH}_{\mathrm{e}}$ values recorded using the sensor give an estimate of the metabolic status of the cells.

The extracellular $\mathrm{pH}\left(\mathrm{pH}_{\mathrm{e}}\right)$ measured using sensor 1 for $2 \times 10^{6}$ cells of PC3, MCF7, DU 145, and normal PBMC $(N=5)$ is depicted in Fig. 2A. Among the cells studied, MCF7 cells displayed the highest acidity with a $\mathrm{pH}$ value of 5.5 followed by DU145 (5.96), PC3 (6.34) and PBMC (7.47). MCF7 cells are known to form intracellular large acidic ( $\mathrm{pH}$ below 4) vesicles (LAVs) which are also found in vitro in breast cancer cells. ${ }^{11}$ The LAVs could probably have some extracellular action resulting in higher acidity in MCF7 as compared to other cancer cells. Using a microelectrode tip, Montcourrier et al. ${ }^{12}$ observed that the free surface of MCF7 has a pH of $0.33 \pm 0.14$ units lower than that of the surrounding medium. But when the microelectrode tip was inserted beneath the attached surface of the cells, the $\mathrm{pH}$ was lowered by up to 1.7 units. This value matches very well with the $\mathrm{pH}$ values estimated for MCF7 using our polyaniline based sensor. The pH value of PC3 measured using sensor 1 (6.34) is slightly lower that the $\mathrm{pH}_{\mathrm{e}}$ value (of 6.83) recently reported by Macholl et al. ${ }^{13}$ using ${ }^{31} \mathrm{P}$ MRS. However, they have indicated that the overall local $\mathrm{pH}_{\mathrm{e}}$ near the cell membrane may be even lower than the $\mathrm{pH}_{\mathrm{e}}$ reported by them as the ${ }^{31} \mathrm{P}$ MRS measured $\mathrm{pH}_{\mathrm{e}}$ is an indicator of the $\mathrm{pH}$ at the site of insertion of the pHLIP membrane on cell membranes. Thus, the $\mathrm{pH}$ values obtained using our sensor assembly give a better estimate of $\mathrm{pH}_{\mathrm{e}}$ and the local microenvironment of the cells as they are in direct contact with the sensor surface. The present electrode assembly can be modified to develop a catheter type insertable electrode for in vivo $\mathrm{pH}_{\mathrm{e}}$ determination at the tumor site, thus reducing the cases of biopsy.

Overall, the measured $\mathrm{pH}_{\mathrm{e}}$ values were found to be much lower for the cancer cells as compared to normal PBM cells. The $\mathrm{pH}$ values reported in the literature for a range of tumors as well as normal cells using a variety of techniques are summarized in Table S2 in the ESI, $\dagger$ where values ranging from 5.5 to 7.6 have been reported. From Fig. 2A, it can be clearly seen that the cancer cells give much higher sensor response as compared to normal cells. Thus, further investigation was carried out to develop a sensor for potential clinical application in detection of cancer.

Sensor 2 with reduced electrode spacing and area (Fig. S1b, ESI $\dagger$ ) was designed so as to allow highly sensitive detection of cancer cells. As discussed earlier, sensing in the present case is a surface phenomenon, where only the cells directly adsorbed on the sensor surface lead to doping of the polyaniline film and thus contribute to the sensor response. In the case of sensor $\mathbf{1}$, the sensor surface area is large to accommodate as high as $1 \times 10^{6}$ cells and hence, the response saturates at cell densities above $1 \times 10^{6}$ cells and is linear below this cell number. To improve the sensitivity of detection, sensor 2 with reduced polyaniline surface area (radius $1 \mathrm{~mm}$ ) was designed so that the surface gets saturated in the presence of a few thousands of cells and shows a linear response for a lower cell number. However, reduction in sensor surface area would lead to very small current output hence, the electrode spacing was reduced to $25 \mu \mathrm{m}$, which helped to obtain high current values. Polyaniline was deposited by placing a $(5 \mu \mathrm{L})$ droplet of the monomer solution over the $25 \mu \mathrm{m}$ electrode spacing followed by electropolymersation to bridge the gap between the electrodes (see details in the ESI, $\dagger$ Section $2 \mathrm{C} 2$ ). The resulting sensor electrode, i.e. sensor 2, gave a linear response in the presence a few numbers of cells as depicted in Fig. $2 \mathrm{~B}$ and displayed high sensitivity for detection of as low as 5 cells (inset of Fig. 2B).

The sensor characteristics for the different cell types studied are compiled in Table S3 in the ESI. $\dagger$ The sensor is generic in nature as no specific antigens or markers were used for recognition. The sensor response was dependent on the nature of metabolism exhibited by the individual cell type. The detection limit of the sensor (estimated at $3 \sigma, \sigma=$ standard deviation of the background signal) was found to be $\sim 0.02 \mathrm{pH}$ units for sensor 1 and $0.002 \mathrm{pH}$ units for sensor 2 . In terms of the cell number, the detection limit was $\sim 4.9 \times 10^{3}$ and 2 MCF7 cells for sensor $\mathbf{1}$ and sensor 2, respectively. The limit of quantification based on measurements using progressively more dilute cell suspension (for sensor 2) was five cells. Studies were also carried out to detect the presence of cancer cells in a cocktail of cancer and normal cells. Sensor 1 could detect $0.01 \times 10^{6}$ MCF7 cells in the presence of $0.5 \times 10^{6}$ normal $\mathrm{CHO}$ cells. This sensitivity for detection of cancer cells in the presence of normal cells can be further increased by using sensor 2 .

Overall, the sensors displayed a low relative standard deviation of less than $3-4 \%(N=5)$, which indicated a good reproducibility of the sensor. As far as the response time is concerned, sensor 1 reached a steady-state current in $\sim 20 \mathrm{~min}$ (sensor $2 \sim 15 \mathrm{~min}$ ) upon addition of an aliquot of cells, thus exhibiting a fairly fast response time as compared to the other traditional methods for cancer detection. Thus, the reported sensor demonstrated satisfactory characteristics when compared with other methods for tumor $\mathrm{pH}$ determination listed in Table S2 in the ESI. $\dagger$

We have further demonstrated the utility of the sensor for screening of drugs used in cancer therapy. Among the various 

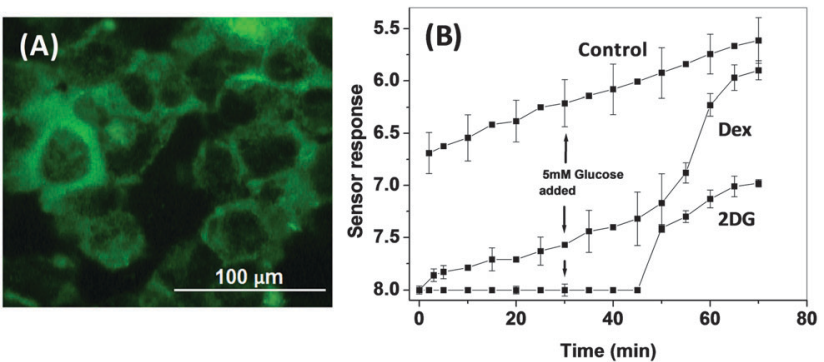

Fig. 3 (A) Fluorescence microscopy image depicting intake of glycolysis inhibiting 2-DG by MCF7 cells in $20 \mathrm{~min}$. (B) Sensor response for real time monitoring of glycolysis inhibition efficiency of drugs dexamethasone (Dex) and 2-deoxy glucose (2-DG) in $2 \times 10^{6}$ MCF7 cells treated with $100 \mu \mathrm{M}$ drug for $24 \mathrm{~h}$, along with control untreated MCF7 cells $(N=3)$. The arrow indicates addition of $5 \mathrm{mM}$ glucose to the sensor.

strategies for developing anti-cancer drugs, glycolysis inhibition is one of the routes followed. The preferential dependence of cancer cells on the glycolytic-pathway for ATP generation provides a biochemical basis for design of therapeutic agents which preferentially kill the cancer cells by inhibition of glycolysis. ${ }^{14}$

Glucocorticoids like dexamethasone (Dex) are most active therapeutic agents in the treatment of leukemia and lymphoid malignancies. ${ }^{15}$ Dex is known to decrease the levels of plasma membrane-associated glucose transporter GLUT1, thus inhibiting glucose uptake. ${ }^{15}$ Another glycolytic inhibitor 2-DG, a glucose analogue, competes with glucose for transmembrane transport (Fig. 3A) and exhibits a cytotoxic effect in cancer cells. ${ }^{14}$ Since our sensor measures $\mathrm{pH}_{\mathrm{e}}$, which is correlated with the generation of metabolic acids via glycolysis, the sensor could be utilized for real time monitoring of the glycolysis inhibiting the efficacy of these drugs at sub-lethal concentrations (see cell viability data in the ESI, $\uparrow$ Section 3F). MCF7 cells treated for $24 \mathrm{~h}$ with either $100 \mu \mathrm{M}$ dexamethasone (Dex) or $100 \mu \mathrm{M}$ 2-deoxyglucose (2DG) exhibited higher $\mathrm{pH}_{\mathrm{e}}$ as compared to untreated cells indicating an efficient glycolysis inhibition by both the drugs (Fig. 3B). Between the two drugs, 2DG exhibited marginally higher glycolysis inhibition (99.8\%) as compared to Dex $(99.72 \%)$ in the absence of glucose in the sensor assembly. Later, $5 \mathrm{mM}$ of glucose was added to the sensor and the inhibition efficiency of the internalized (cell entrapped) drug in the presence of glucose was studied. After 20 min of addition of glucose, both the drug treated MCF7 cells started showing signs of commencement of glycolysis. However, the internalized 2DG was able to inhibit glycolysis to a higher extent as compared to Dex $(98.5 \%$ and $88.3 \%$ inhibition, respectively, $30 \mathrm{~min}$ after addition of glucose), indicating better glycolysis inhibition efficacy of 2DG as compared to Dex. The results are consistent with the reports of Buentke et al.,${ }^{15}$ where inhibition efficiency was studied by assessing the metabolites by liquid chromatography and mass spectrometry.

To summarize, we have devised a novel multifunctional platform having three fold potential application in: (a) biological research for determination of extracellular acidity i.e. $\mathrm{pH}_{\mathrm{e}}$ in real time, (b) diagnosis for highly sensitive detection of cancer cells, and (c) drug discovery for evaluating the efficiency of glycolysis inhibiting anti-cancer drugs in real time.

Another advantage of using a polyaniline-based sensor is its biocompatibility, which we have demonstrated in one of our earlier studies. ${ }^{16}$ Thus, the sensor film will not alter the physiological functioning of the cells and can be utilized for in vivo spatiotemporal measurements of extracellular $\mathrm{pH}$. The novel concept demonstrated herein opens a way, not only for preliminary screening and diagnosis of cancer, but also in cancer metabonomics for monitoring the response to various therapies during the treatment of cancer.

BT is thankful to the BARC-UoP collaborative PhD program for the fellowship.

\section{Notes and references}

1 U. Meyer, T. Meyer, J. Handschel and H. P. Wiesmann, Fundamentals of Tissue Engineering and Regenerative Medicine, Springer-Verlag, Berlin, Heidelberg, 2009, p. 79.

2 X. Li, Y. Pei, R. Zhang, Q. Shuai, F. Wang, T. Aastrup and Z. Pie, Chem. Commun., 2013, 49, 9908; S. Krishnan, E. G. Hvastkovs, B. Bajrami, I. Jansson, J. B. Schenkman and J. F. Rusling, Chem. Commun., 2007, 1713; J. Liu, C.-Y. Lu, H. Zhou, J.-J. Xu, Z.-H. Wang and H.-Y. Chen, Chem. Commun., 2013, 49, 6602; P. Chandra, H.-B. Noh and Y.-B. Shim, Chem. Commun., 2013, 49, 1900.

3 J. W. Wojtkowiak, J. M. Rothberg, V. Kumar, K. J. Schramm, E. J. Haller, B. Proemsey, M. C. Lloyd, B. F. Sloane and R. J. Gillies, Cancer Res., 2012, 72, 3938.

4 M. F. MacCarty and J. Whitaker, Altern. Med. Rev., 2010, 15, 264.

5 A. Schulze and A. L. Harris, Nature, 2012, 491, 364-373.

6 L. E. Gerweck, S. Vijayappa and S. Kozin, Mol. Cancer Ther., 2006, $5,1275$.

7 D. Lindner and D. Raghavan, Br. J. Cancer, 2009, 100, 1287.

8 F. Kallinowski and P. Vaupel, Br. J. Cancer, 1988, 58, 314.

9 M. U. A. Prathap, A. K. Chaurasia, S. N. Sawant and S. K. Apte, Anal. Chem., 2012, 84, 6672.

10 S. K. Arya, K. C. Lee, D. B. Dah'alan, Daniel and A. R. A. Rahman, Lab Chip, 2012, 12, 2362-2368.

11 P. Montcourrier, P. H. Mangeat, C. Valembois, G. Salazar, A. Sahuquet, C. Duperray and H. Rochefort, J. Cell Sci., 1994, 107, 2381.

12 P. Montcourrier, I. Silver, R. Farnoud, I. Bird and H. Rochefort, Clin. Exp. Metastasis, 1997, 15, 382.

13 S. Macholl, M. S. Morrison, P. Iveson, B. E. Arbo, O. A. Andreev, Y. K. Reshetnyak, D. M. Engelman and E. Johannesen, Mol. Imaging Biol., 2012, 14, 725.

14 H. Pelicano, D. S. Martin, R.-H. Xu and P. Huang, Oncogene, 2006, 25, 4633.

15 E. Buentke, A. Nordström, H. Lin, A. C. Björklund, E. Laane, M. Harada, L. Lu, T. Tegnebratt, S. Stone-Elander, M. Heyman, S. Söderha, A. Porwit, C. G. Östenson, M. Shoshan, K. P. Tamm and D. Grandér, Blood Cancer Journal, 2011, 1, e31.

16 P. K. Prabhakar, S. Raj, P. R. Anuradha, S. N. Sawant and M. Doble, Colloids Surf., B, 2011, 86, 146. 